Pacific Journal of Mathematics

ON IDEALS IN $\Omega_{*}^{u}$ 


\section{ON IDEALS IN $\Omega_{*}^{V}$}

\section{LARRY SMITH}

The objective of these notes is to study the relations between and the structure of various ideals that occur in the study of the complex bordism homology functor and actions of abelian $p$-groups on closed weakly complex manifolds.

Let us fix a prime integer $p$ and denote by $\boldsymbol{K}\left(\boldsymbol{Z}_{p}, n\right)$ an EilenbergMacLane space of type $\left(\boldsymbol{Z}_{p}, n\right)$. Let

$$
f: S^{n} \longrightarrow \boldsymbol{K}\left(\boldsymbol{Z}_{p}, n\right)
$$

represent a generator of $\pi_{n}\left(\boldsymbol{K}\left(\boldsymbol{Z}_{p}, n\right)\right)=\boldsymbol{Z}_{p}$ and set

$$
\sigma_{n}=\left[S^{n}, f\right] \in \Omega_{n}^{U}\left(\boldsymbol{K}\left(\boldsymbol{Z}_{p}, n\right)\right) .
$$

We propose to study the annihilator ideal, $A\left(\sigma_{n}\right) \subset \Omega_{*}^{U}$, of the $U$-bordism class $\sigma_{n}$. To this end let us denote by $K$ the ideal which is the kernel of the natural map

$$
\Phi_{(p)}: Q_{*}^{U} \longrightarrow H_{*}\left(B U ; \boldsymbol{Z}_{p}\right) \text {. }
$$

Thus the elements of $K$ are those $U$-manifolds all of whose Chern numbers are congruent to zero $\bmod p$. The structure of the ideal $K$ is known as a consequence of the work of Milnor [8]. Namely, for each nonnegative integer $i$, there exists a "Milnor" manifold $V^{2 p^{i}-2}$, of dimension $2 p^{i}-2$, such that

$$
K=\left(\left[V^{0}\right],\left[V^{2 p-2}\right], \cdots\right) .
$$

The first elementary fact concerning the ideal $A\left(\sigma_{n}\right)$ is the inclusion $A\left(\sigma_{n}\right) \subset K$. Thus it makes sense to inquire into which of the Milnor manifolds [ $\left.V^{0}\right],\left[V^{2 p-2}\right], \cdots$, actually lie in $A\left(\sigma_{n}\right)$. One of our objectives in this note is to establish the following two results.

THEOREM A: $A\left(\sigma_{n}\right) \ni p,\left[V^{2 p-2}\right], \cdots,\left[V^{2 p^{n-1}-2}\right]$.

Theorem B: $A\left(\sigma_{n}\right) \nexists\left[V^{2 p^{n}-2}\right], \cdots,\left[V^{2 p^{n+m}-2}\right], \cdots$.

Thus we determine completely which of the Milnor manifolds annihilate the class $\sigma_{n}$. While this does not determine the structure of $A\left(\sigma_{n}\right)$ it is a step towards that goal. The study of annihilator ideals of spherical bordism classes has been a recurrent theme throughout the investigations [5], [6], [10], [11] of the complex bordism of finite complexes. The ideal $A\left(\sigma_{n}\right)$ is in an appropriate sense a universal example of such an ideal. 
To connect up the study of the ideals $A\left(\sigma_{n}\right)$ with the study of free actions of abelian $p$-groups on closed weakly complex manifolds we introduce the infinite dimensional lens space $\boldsymbol{L}\left(\boldsymbol{Z}_{p}, \infty\right)=\boldsymbol{K}\left(\boldsymbol{Z}_{p}, 1\right)=$ $B Z_{p}$ that classifies principal $Z_{p}$ bundles. (For $p=2$ this may be taken to be the infinite dimensional real projective space.) We let $\gamma \in \widetilde{\Omega}_{1}^{L}\left(B \boldsymbol{Z}_{p}\right)$ denote the class represented by the canonical map $S^{1} \rightarrow$ $B Z_{p}$. The exterior product

$$
\underbrace{S^{1} \times \cdots \times S^{1}}_{n \text {-fois }} \longrightarrow B \underbrace{Z_{p} \times \cdots \times B Z_{p}}_{n \text {-fois }}
$$

represents an $n$-dimensional bordism class that we denote by $\otimes^{n} \gamma \in$ $\widetilde{\Omega}_{n}^{v}\left(B \boldsymbol{Z}_{p}^{n}\right)$. With these notations fixed we introduce a trio of ideals:

$$
\begin{aligned}
& A_{n}= A\left(\mathbb{Q}^{n} \gamma\right)=\left\{[M] \in \Omega_{*}^{U} \mid[M] \otimes^{n} \gamma=0 \in \widetilde{\Omega}_{*}^{U}\left(B Z_{p}^{n}\right)\right\} \\
& I_{n}: \text { defined inductively by } \\
& I_{0}=(0) \\
& I_{n}=\left\{[M] \in \Omega_{*}^{U} \mid[M] \gamma \in I_{n-1} \widetilde{\Omega}_{*}^{U}\left(B \boldsymbol{Z}_{p}\right)\right\} \\
& J_{n}=\left\{\begin{array}{l}
{[M] \in \Omega_{*}^{U} \mid \text { there exists a representative }} \\
M \text { of }[M] \text { upon which } Z_{p} \underset{(n \text {-fois })}{\ldots} \times Z_{p} \text { acts } \\
\text { without stationary points } .
\end{array}\right\}
\end{aligned}
$$

(Note that each of these ideals depends on the prime $p$ but that we have supressed the dependence from our notation.) The ideals $J_{n}$ were introduced in [2] where the study of their structure was first undertaken. In [6] E. E. Floyd computes both the ideals $I_{n}$ and $J_{n}$ and shows that they coincide.

Among the elementary facts that one has is that each of the above families of ideals forms an expanding sequence, viz.

$$
\begin{aligned}
& (0)=A_{0} \subset A_{1} \subset A_{2} \subset \cdots \\
& (0)=S_{0} \subset S_{1} \subset S_{2} \subset \cdots \\
& (0)=I_{0} \subset I_{1} \subset I_{2} \subset \cdots \\
& (0)=J_{0} \subset J_{1} \subset J_{2} \subset \cdots
\end{aligned}
$$

where we have written $S_{n}$ for $A\left(\sigma_{n}\right)$. This suggests that we introduce the stable ideals

$$
\begin{gathered}
A=\bigcup_{n=0}^{\infty} A_{n}, S=\bigcup_{n=0}^{\infty} S_{n} \\
I=\bigcup_{n=0}^{\infty} I_{n}, J=\bigcup_{n=0}^{\infty} J_{n} .
\end{gathered}
$$

The results relating all these ideals that we shall establish are 
Theorem C: $I_{n}=J_{n} \subset A_{n} \subset S_{n}$.

Theorem D: $I=J=A=S=K$.

It is to be emphasized that the structure theorem of Floyd [6] for the ideals $I_{n}$ and $J_{n}$ essentially contains all the information that we require to establish Theorems A, C, and D. Our main contribution is Theorem B. It follows from Floyd's work that

$$
I_{n}=\left(\left[V^{0}\right],\left[V^{2 p-2}\right], \cdots,\left[V^{2 p^{n-1}-2}\right]\right)=J_{n}
$$

and hence Theorem $\mathrm{A}$ is an instant consequence of Theorem C. Thus (i.e. from [6]) we see that

$$
I=\left(\left[V^{0}\right],\left[V^{2 p-2}\right], \cdots,\left[V^{2 p^{m}-2}\right], \cdots\right)=J .
$$

As noted previously the work of Milnor [8] shows

$$
K=\left(\left[V^{0}\right],\left[V^{2 p-2}\right], \cdots,\left[V^{2 p^{m}-2}\right], \cdots\right) .
$$

and thus Theorem $\mathrm{D}$ follows from the equality $S=K$ (or the observation that $S_{n} \subset K$ for all $n \geqq 0$ ) and Theorem C. The remainder of these notes is devoted to the proof of Theorem $\mathrm{B}$ and the verification of the necessary inclusion relations between the various ideals required to prove Theorem $\mathrm{C}$ and complete the proof of Theorem $\mathrm{D}$.

1. Preliminaries. The proof of Theorem $B$ requires that we review in some detail certain results of [5]. To this end let us denote by $M$ a closed weakly complex manifold. We continue to denote by $p$ a prime and we let

$$
s_{p^{i}-1}(c) \in H^{2 p^{i-2}}\left(M ; Z_{p}\right)
$$

denote the usual $s_{\omega}$-symmetric function of the Chern classes of $M$ [3] [12]. Our purposes require that we have available a procedure for evaluating the number

$$
\left\langle a \cup s_{p^{i}-1}(c),[M]\right\rangle \in \boldsymbol{Z}_{p} ; a \in H^{*}\left(M, Z_{p}\right),
$$

in terms of "other" invariants of $M$. The required procedure consists of a $W u$ type formula established in [5]. To describe this formula we must collect some elementary facts concerning the Steenrod algebra [7].

Recollections and Notations. Let $\mathscr{A}^{*}(p)$ denote the mod $p$ Steenrod algebra and $\mathscr{P}^{*}(p)$ the algebra of reduced power operations, i.e.,

$$
\mathscr{P}^{*}(p)=\mathscr{A}^{*}(p) /(\beta)
$$


where $(\beta)$ denotes the two sided ideal generated by $\beta$. (Recall that $\beta=S q^{1}$ when $p=2$.)

According to Milnor [7] the dual Hopf algebra $\mathscr{S}_{*}(p)$ is given by

$$
\mathscr{P}_{*}(p)=\boldsymbol{Z}_{p}\left[\left\{\mu_{i}\right\}\right]
$$

where

$$
\operatorname{deg} \mu_{i}=2\left(p^{i}-1\right)
$$

and

$$
\nabla^{*} \mu_{k}=\sum_{i=0}^{k} \mu_{k-i}^{p^{i}} \otimes \mu_{i}
$$

with the convention that $\mu_{0}=1$.

The duals of the classes $\left\{\mu_{i}\right\}$ are primitive elements of $\mathscr{P}^{*}(p)$ which may be defined inductively by the formulas

$$
S_{i}=\left\{\begin{array}{l}
P_{p}^{1} ; i=1, \\
{\left[S_{i-1}, p_{p}^{p i-1}\right] ; i>1 .}
\end{array}\right.
$$

These formulas determine corresponding unique elements $S_{i} \in \mathscr{A}^{*}(p)$ of degree $2\left(p^{i}-1\right)$ which are primitive provided that $p \neq 2$. (Recall that for $p=2, P_{2}^{1}=S q^{2}$.)

The following result may be found in $[5 ; 1.2]$.

THEOREM 1.1. Let $M^{m}$ be a closed weakly complex manifold of dimension $m$ and $a \in H^{m-2\left(p^{i}-1\right)}\left(M ; \boldsymbol{Z}_{p}\right)$. Then with the preceeding notations we have

$$
\left\langle a \cup s_{p} i_{-1}(c),[M]\right\rangle=\left\langle S_{i} a,[M]\right\rangle \in \boldsymbol{Z}_{p}
$$

at least up to a unit in $\boldsymbol{Z}_{p}$. **

As an indication of how we intend to apply this theorem let us suppose that we are given a closed $U$-manifold $M^{m}$ and a map

$$
f: M^{m} \longrightarrow S^{k}: m-k=2\left(p^{i}-1\right),
$$

and a cohomology class $a \in H^{k}(M ; Z)$ such that

$$
f^{*}(\iota)=p a
$$

where $\iota \in H^{k}\left(S^{k} ; \boldsymbol{Z}\right)$ is the fundamental class. Then according to Milnor [8] and an elementary transverse regularity argument

$$
[M, f]=\left[V^{2 p^{i}-2}\right] \sigma \in \widetilde{\Omega}_{*}^{U}\left(S^{k}\right)
$$

(where $\sigma \in \widetilde{\Omega}_{k}^{v}\left(S^{k}\right)$ is the fundamental bordism class) if and only if

$$
\left\langle p a \cup s_{p^{i-1}}(c),[M]\right\rangle \equiv p \bmod p^{2} .
$$


Now clearly this latter condition can hold if and only if

$$
\left\langle a \cup s_{p^{i}-1}(c),[M]\right\rangle \neq 0 \bmod p .
$$

In view of Poincare duality and Theorem 1.1 we thus obtain:

THEOREM 1.2. Suppose given a closed weakly complex manifold $M^{m}$ and a map

$$
f: M^{m} \longrightarrow S^{k}: m-k=2 p^{i}-2,
$$

together with a cohomology class $a \in H^{k}(M ; Z)$ such that

$$
f^{*}(\iota)=p a \text {. }
$$

Then

$$
[M, f]=\left[V^{2 p^{i}-2}\right] \sigma \in \widetilde{\Omega}_{*}^{U}\left(S^{k}\right)
$$

if and only if

$$
\left\langle S_{i} a,[M]\right\rangle \neq 0 \in Z_{p}
$$

i.e., if and only if

$$
S_{i}(a) \neq 0 \in H^{m}\left(M^{m} ; \boldsymbol{Z}_{p}\right)
$$

where we have written a for its own mod $p$ reduction.

2. The proof of Theorem B. We continue to denote by

$$
f: S^{n} \longrightarrow \boldsymbol{K}\left(\boldsymbol{Z}_{p}, n\right)
$$

a map representing a generator of $\pi_{n}\left(\mathbb{K}\left(\boldsymbol{Z}_{n}, n\right)\right) \cong \boldsymbol{Z}_{n}$. Let us introduce the cofibration

$$
\boldsymbol{K}\left(\boldsymbol{Z}_{p}, n\right) \underset{h}{\longrightarrow} \boldsymbol{K}\left(\boldsymbol{Z}_{p}, h\right) \bigcup_{f} e^{n+1} \underset{g}{\longrightarrow} S^{n+1} .
$$

We then have the following elementary result:

Proposition 2.1. With the notations as above we have

$$
[M] \in A\left(\sigma_{n}\right)
$$

if and only if

$$
[M] i_{n+1} \in \operatorname{Im}\left\{g_{*}: \widetilde{\Omega}_{*}^{U}\left(\boldsymbol{K}\left(\boldsymbol{Z}_{p},, n\right) \bigcup_{f} e^{n+1}\right) \longrightarrow \widetilde{\Omega}_{*}^{U}\left(S^{n+1}\right)\right\},
$$

where

$$
i_{n+1}=\left[S^{n+1}, i d\right] \in \widetilde{\Omega}_{n+1}^{U}\left(S^{n+1}\right)
$$

is the canonical class.

Proof. This results from the exact triangle 


$$
\widetilde{\Omega}_{*}^{U}\left(\boldsymbol{K}\left(\boldsymbol{Z}_{p}, n\right)\right) \underset{\partial_{*} \backslash}{h_{*}} \overbrace{g_{*}}^{\longrightarrow} \widetilde{\Omega}_{*}^{U}\left(\boldsymbol{K}\left(\boldsymbol{Z}_{p}, n\right) \mathbf{U}_{f} e^{n+1}\right)
$$

upon recalling that $\partial_{*} i_{n+1}=\left[S^{n}, f\right]=\sigma_{n} \cdot \square$

Notice the elementary fact that the sequence

$$
\begin{aligned}
0=H^{n+2}\left(S^{n+1} ; \boldsymbol{Z}\right) & \longleftarrow H^{n+1}\left(\boldsymbol{K}\left(\boldsymbol{Z}_{p}, n\right) ; \boldsymbol{Z}\right) \\
& \longleftarrow H^{n+1}\left(\boldsymbol{K}\left(\boldsymbol{Z}_{p}, n\right) \bigcup_{f} e^{n+1} ; \boldsymbol{Z}\right) \longleftarrow H^{n+1}\left(S^{n+1} ; \boldsymbol{Z}\right) \\
& \longleftarrow H^{n}\left(\boldsymbol{K}\left(\boldsymbol{Z}_{p}, n\right) ; \boldsymbol{Z}\right)=0
\end{aligned}
$$

is exact, not split, and has

$$
\begin{array}{r}
H^{n+1}\left(S^{n+1} ; \boldsymbol{Z}\right) \cong \boldsymbol{Z} \cong H^{n+1}\left(\boldsymbol{K}\left(\boldsymbol{Z}_{p}, n\right) \bigcup_{f} e^{n+1} ; \boldsymbol{Z}\right) \\
H^{n+1}\left(\boldsymbol{K}\left(\boldsymbol{Z}_{p}, n\right) ; \boldsymbol{Z}\right) \cong \boldsymbol{Z}_{p} .
\end{array}
$$

Therefore for an appropriate choice of generator

we have

$$
e_{n+1} \in H^{n+1}\left(\boldsymbol{K}\left(\boldsymbol{Z}_{p}, n\right) \bigcup_{f} e^{n+1} ; \boldsymbol{Z}\right)
$$

$$
g^{*}(\iota)=p e_{n+1} .
$$

With these notations fixed we are now prepared to take up the proof of Theorem B.

Proof of Theorem B. Let us suppose that

$$
\left[V^{2 p^{i}-2}\right] \in A\left(\sigma_{n}\right) \text {. }
$$

Then according to, and in the notation of, Proposition 2.1 we must have

$$
\left[V^{2 p^{i}-2}\right] i_{n+1} \in \operatorname{Im}\left\{g_{*}: \widetilde{\Omega}_{*}^{U}\left(\boldsymbol{K}\left(\boldsymbol{Z}_{p}, n\right) \mathbf{U}_{f} e^{n+1}\right) \longrightarrow \widetilde{\Omega}_{*}^{U}\left(S^{n+1}\right)\right\} .
$$

Let $[M, \varphi] \in \widetilde{\Omega}_{*}^{v}\left(\boldsymbol{K}\left(\boldsymbol{Z}_{p}, n\right) \bigcup_{f} e^{n+1}\right)$ be chosen with

Then of course

$$
g_{*}[M, \varnothing]=\left[V^{2 p^{i}-2}\right] i_{n+1} \text {. }
$$

Recall that

$$
[M, g \varphi]=\left[V^{2 p^{i}-2}\right] i_{n+1} .
$$

Thus by setting

$$
g^{*}(\iota)=p e_{n+1} .
$$

$$
a=\varphi^{*}\left(e_{n+1}\right) \in H^{*}(M ; \boldsymbol{Z})
$$

we find that with this cohomology class and the map

$$
g \varphi: M \longrightarrow S^{n+1}
$$


we may apply Theorem 1.2 to conclude that

$$
\left\langle S_{i} a,[M]\right\rangle \neq 0 \bmod p .
$$

Of course by naturality

$$
\left\langle S_{i} a,[M]\right\rangle=\left\langle\varphi^{*} S_{i} e_{n+1},[M]\right\rangle
$$

(where we have written $e_{n+1}$ for its own $\bmod p$ reduction). Now recall that the map

$$
h^{*}: H^{*}\left(K\left(\boldsymbol{Z}_{p}, n\right) \bigcup_{f} e^{n+1} ; \boldsymbol{Z}_{p}\right) \longrightarrow H^{*}\left(K\left(\boldsymbol{Z}_{p}, n\right), \boldsymbol{Z}_{p}\right)
$$

is an isomorphism for $*>n+1$. As we have already observed that $p=\left[V^{0}\right] \in A\left(\sigma_{n}\right)$ we may as well assume that $i>0$. It is now time to note that $h^{*}\left(e_{n+1}\right)=\beta \theta_{n}$, where $\theta_{n} \in H^{n}\left(K\left(\boldsymbol{Z}_{p}, n\right) ; \boldsymbol{Z}_{p}\right)$ is the fundamental class. Thus

$$
h * S_{i} e_{n+1}=S_{i} \beta \theta_{n} .
$$

Suppose now that $i>n$. Examination of the results of Cartan [1] and Serre [9] reveal that for $\mathrm{i}>n$

$$
S_{i} \beta \theta_{n}=\rho v
$$

where $\rho$ is reduction $\bmod p$ and $v \in H^{*}\left(K\left(\boldsymbol{Z}_{p}, n\right) ; \boldsymbol{Z}\right)$ an integral class. Let $u \in H\left(K\left(\boldsymbol{Z}_{p}, n\right) \mathbf{U}_{f} e^{n+1} ; \boldsymbol{Z}\right)$ be an integral class with $h^{*} u=v$. Thus we have found

$$
\left[V^{2 p^{i}-2}\right] \in A\left(\sigma_{n}\right) \Longrightarrow\left\langle\varphi^{*} S_{i} e_{n+1},[M]\right\rangle \neq 0 .
$$

Since $i>n$ the preceeding discussion shows

$$
\begin{aligned}
\left\langle\varphi^{*} S_{i} e_{n+1},[M]\right\rangle & =\left\langle\varphi^{*} \rho u,[M]\right\rangle \\
& =\left\langle\rho \varphi^{*} u,[M]\right\rangle
\end{aligned}
$$

and since $[M]$ is an integral class

$$
\begin{aligned}
\left\langle\varphi^{*} S_{i} e_{n+1},[M]\right\rangle & =\rho\left\langle\varphi^{*} u,[M]\right\rangle \\
& =\rho\left\langle u, \varphi^{*}[M]\right\rangle .
\end{aligned}
$$

However, since $H_{*}\left(K\left(\boldsymbol{Z}_{p}, n\right) \bigcup_{f} e^{n+1} ; \boldsymbol{Z}\right)$ is all torsion for ${ }^{*}>n+1$ it follows that the Kronecker index over the integers $\boldsymbol{Z}$ for $K\left(\boldsymbol{Z}_{p}, n\right) \bigcup_{f} e^{n+1}$ is always zero for ${ }^{*}>n+1$. Therefore

$$
\left\langle u, \varphi_{*}[M]\right\rangle=0
$$

and hence

$$
\left\langle\varphi^{*} S_{i} e_{n+1},[M]\right\rangle=0
$$

contrary to our previous calculation. This contradiction shows that 
[ $V^{2 p^{i}-2}$ ] cannot belong to the ideal $A\left(\sigma_{n}\right)$ for any $i>n$ which is the desired conclusion.

3. Various inclusions. We collect here in this section the various inclusion relations required to establish Theorem $\mathrm{C}$ and to complete the proof of Theorem D.

The Inclusion $A_{n} \subset A_{n+1}$. Consider the exterior cross product

$$
\widetilde{\Omega}_{*}^{U}\left(B \boldsymbol{Z}_{p}^{n}\right) \otimes_{\Omega_{*}^{U}} \widetilde{\Omega}_{*}^{U}\left(B \boldsymbol{Z}_{p}\right) \longrightarrow \widetilde{\Omega}_{*}^{U}\left(B \boldsymbol{Z}_{p}^{n+1}\right) \text {. }
$$

Let us suppose that $[M] \in A_{n}$, then by naturality of the cross-product we find

$$
[M] \otimes^{n+1} \gamma=[M] \otimes^{n} \gamma \otimes \gamma=0 \otimes \gamma=0
$$

and hence $[M] \in A_{n+1}$ which is the desired conclusion.

The Inclusion $S_{n} \subset S_{n+1}$. Introduce the map

$$
u_{n}: \Sigma K\left(Z_{p}, n\right) \longrightarrow K\left(Z_{p}, n+1\right)
$$

that classifies the cohomology class

$$
\Sigma \theta_{n} \in H^{n+1}\left(\boldsymbol{K}\left(\boldsymbol{Z}_{p}, n\right) ; \boldsymbol{Z}_{p}\right) \text {. }
$$

It is easily checked that

$$
\left(u_{n}\right)_{*}\left(\Sigma \sigma_{n}\right)=\sigma_{n+1} .
$$

Thus if $[M] \in A\left(\sigma_{n}\right)=S_{n}$ then by naturality and stability under suspension we find

$$
[M] \sigma_{n+1}=\left(u_{n}\right)_{*}\left(\Sigma[M] \sigma_{n}\right)=0
$$

and hence $[M] \in S_{n+1}$ as required.

The Equality $S=K$. Consider the morphism of spectra

$$
v: S \longrightarrow K\left(Z_{p}\right)
$$

that defines the unit of the latter. Its component morphisms are the maps

$$
f: S^{n} \longrightarrow K\left(Z_{p}, n\right): n=0,1,2, \cdots
$$

and as

$$
S_{n}=\operatorname{ker}\left\{f_{*}: \widetilde{\Omega}_{*}^{U}\left(S^{n}\right) \longrightarrow \widetilde{\Omega}_{*}^{U}\left(\boldsymbol{K}\left(\boldsymbol{Z}_{p}, n\right)\right)\right\}
$$

we find

$$
S=\cup S_{n}=\operatorname{ker}\left\{v_{*}: \widetilde{\Omega}_{*}^{U}(\boldsymbol{S}) \longrightarrow \widetilde{\Omega}_{*}^{U}\left(\boldsymbol{K}\left(\boldsymbol{Z}_{p}\right)\right)\right\}
$$


since both $\boldsymbol{S}$ and $\boldsymbol{K}\left(\boldsymbol{Z}_{p}\right)$ are convergent spectra.

Now ker $v_{*}$ may be given another interpretation as follows. Recall that

$$
\begin{gathered}
\widetilde{\Omega}_{*}^{U}\left(\boldsymbol{K}\left(\boldsymbol{Z}_{p}\right)\right)=\pi_{*}^{s}\left(\boldsymbol{M U} \wedge \boldsymbol{K}\left(\boldsymbol{Z}_{p}\right)\right)=\widetilde{H}_{*}\left(\boldsymbol{M U} ; \boldsymbol{Z}_{p}\right) \\
\widetilde{\Omega}_{*}^{U}(\boldsymbol{S})=\Omega_{*}^{U},
\end{gathered}
$$

and under the identifications above we may view $v_{*}$ as the natural map

$$
\dot{\phi}_{(p)}=v_{*}: \Omega_{*}^{*} \longrightarrow \widetilde{H}_{*}\left(\boldsymbol{M U} ; \boldsymbol{Z}_{p}\right)
$$

which by the Thom isomorphism may be viewed as

$$
\Phi_{(p)}=v_{*}: \Omega_{*}^{U} \longrightarrow \widetilde{H}_{*}\left(B U ; \boldsymbol{Z}_{p}\right)
$$

where

$$
\left\langle v_{*}[M], c_{\omega}\right\rangle=c_{\omega}[M]
$$

for any monomial $c_{\omega} \in H^{*}\left(B U ; \boldsymbol{Z}_{p}\right)$ in the $\bmod p$ Chern classes. As

$$
\langle,\rangle: H_{*}\left(B U ; \boldsymbol{Z}_{p}\right) \otimes H^{*}\left(B U: \boldsymbol{Z}_{p}\right) \longrightarrow \boldsymbol{Z}_{p}
$$

is a dual pairing we find

$$
\operatorname{ker} v_{*}=\left\{[M] \in \Omega_{*}^{J} \mid c_{\omega}[M] \equiv 0 \bmod p: \text { all } \omega\right\}=K
$$

and the desired equality follows.

The Inclusion $I_{n} \subset A_{n}$. Let us consider first the case $n=1$. We find

$$
I_{1}=\left\{[M] \in \Omega_{*}^{\prime r} \mid[M] \gamma=0 \in Q_{*}^{V}\left(B \boldsymbol{Z}_{p}\right)\right\}=A_{1} .
$$

Thus $I_{1} \subseteq A_{1}$. We may therefore proceed inductively and assume that $I_{m} \subseteq A_{m}$ for all $m<n$. We wish to conclude $I_{n} \subseteq A_{n}$. We may as well assume that $n>1$. Accordingly we consider the exterior product

$$
\Omega_{*}^{U}\left(B \boldsymbol{Z}_{p}^{n-1}\right) \bigotimes_{2_{*}^{U}} \Omega_{*}^{U}\left(B \boldsymbol{Z}_{p}\right) \longrightarrow \Omega_{*}^{U}\left(B \boldsymbol{Z}_{p}^{n}\right) .
$$

One readily sees that with the obvious abuse of notation that

$$
\otimes^{n-1} \gamma \otimes \gamma=\otimes^{n} \gamma
$$

in $\Omega_{*}^{y}\left(B \boldsymbol{Z}_{p}^{n}\right)$. Suppose that $[M] \in I_{n}$. Then we have

$$
[M] \gamma \in I_{n-1} Q_{*}^{U}\left(B Z_{p}\right)
$$

and thus

$$
[M]=\Sigma\left[N_{i}\right] \lambda_{i}
$$

where

$$
\left[N_{i}\right] \in I_{n-1}, \lambda_{i} \in \widetilde{\Omega}_{*}^{c}\left(B Z_{p}\right)
$$


Thus we find

$$
\begin{aligned}
{[M] \bigotimes^{n} \gamma } & =\bigotimes^{n-1} \gamma \otimes[M] \gamma=\bigotimes^{n-1} \gamma \otimes \Sigma\left[N_{i}\right] \lambda_{i} \\
& =\Sigma\left[N_{i}\right] \bigotimes^{n-1} \gamma \otimes \lambda_{i}
\end{aligned}
$$

by naturality. By our inductive assumption $I_{n-1} \subseteq A_{n-1}$ and hence

$$
\left[N_{i}\right] \bigotimes^{n-1} \gamma=0 \in \Omega_{*}^{U}\left(B Z_{p}^{n-1}\right) .
$$

Therefore we find

$$
[M] \otimes^{n} \gamma=\Sigma\left[N_{i}\right] \otimes^{n-1} \gamma \otimes \lambda_{i}=\Sigma 0 \otimes \lambda_{i}=0
$$

and hence $[M] \in A_{n}$ as was to be shown. Thus the inclusion $I_{n} \subset A_{n}$ is established inductively for all $n \geqq 0$.

The Inclusion $A_{n} \subset S_{n}$. Consider the standard map

$$
q_{n}: B \boldsymbol{Z}_{p}^{n} \longrightarrow \boldsymbol{K}\left(\boldsymbol{Z}_{p}, n\right)
$$

that classifies the cohomology class

$$
\theta_{1} \otimes \theta_{1} \otimes \cdots \otimes \theta_{1} \in H^{n}\left(B \boldsymbol{Z}_{p}^{n} ; \boldsymbol{Z}_{p}\right)
$$

It is easily checked that

$$
\left(q_{n}\right)_{*}\left(\otimes^{n} \gamma\right)=\sigma_{n} .
$$

Suppose now that $[M] \in A_{n}$. Then by naturality we find

$$
[M] \sigma_{n}=\left(q_{n}\right)_{*}[M] \otimes^{n} \gamma=0
$$

and therefore $[M] \in S_{n}$ as desired.

Closing Remarks. For $n=1$ and 2 it may be shown by brute force computational techniques that

$$
I_{n}=A_{n}=S_{n}: n=1,2 .
$$

In view of the work of Floyd [6] the structure of all these ideals are then known. We conjecture more generally that this equality holds for all $n \geqq 0$, and in particular that

$$
A_{n}=\left(p,\left[V^{2 p-2}\right], \cdots,\left[V^{2 p^{n-1}-2}\right]\right)=S_{n} .
$$

\section{REFERENCES}

1. H. Cartan, Algebres d'Eilenberg-MacLane et Homotopie, Seminar H. Cartan 1954/55, E.N.S. Paris.

2. P. E. Conner and E. E. Floyd, Differentiable Periodic Maps, Springer-Verlag, New York, 1964. 
3. The Relation of Cobordism Theories to K-Theories, Springer Lecture Notes in Math. No. 28, 1966.

4. P. E. Conner and L. Smith, On the Complex Bordism of Finite Complexes, I.H.E.S. Journal de Math., No. 37, (1970), 117-221.

5. - On the Complex Bordism of Finite Complexes II, U.VA. Preprint 1970.

6. E. E. Floyd, Actions of $\boldsymbol{Z}_{p}^{k}$ Without Stationary Points, (to appear).

7. J. W. Milnor, On the Steenrod Algebra and its Dual, Annals of Math., 67 (1958), $150-171$.

8. - On the Cobordism Ring $\Omega^{*}$ and a Complex Analog, Amer. J. Math., 82 (1960), 505-521.

9. J-P. Serre, Cohomologie modulo deux des complexes d'Eilenberg-MacLane, Comm. Math. Helv., 27 (1953), 198-231.

10. L. Smith, An Application of Complex bordism to the stable homotopy groups of spheres, Bull. Amer. Math. Soc., 76 (1970), 601-604.

11. - On Realizing Complex Bordism Modules, Amer. J. Math., 92 (1970), $793-856$.

12. R. E. Stong, Notes on Cobordism Theory, Princeton University Press, 1968.

Received May 4, 1970.

Institute des Hautes Études Scientifiques, 91-Bures-sur-Yvette, France AND

THE UNIVERSity OF Virginia 



\section{PACIFIC JOURNAL OF MATHEMATICS}

\section{EDITORS}

\author{
H. SAMElson \\ Stanford University \\ Stanford, California 94305 \\ C. R. HOBBY \\ University of Washington \\ Seattle, Washington 98105
}

J. DugundjI

Department of Mathematics University of Southern California Los Angeles, California 90007

RICHARD ARENS

University of California

Los Angeles, California 90024

\section{ASSOCIATE EDITORS}
E. F. BECKENBACH
B. H. NeUmanN
F. WOLE
K. YOSHIDA

\section{SUPPORTING INSTITUTIONS}

\author{
UNIVERSITY OF BRITISH COLUMBIA \\ CALIFORNIA INSTITUTE OF TECHNOLOGY \\ UNIVERSITY OF CALIFORNIA \\ MONTANA STATE UNIVERSITY \\ UNIVERSITY OF NEVADA \\ NEW MEXICO STATE UNIVERSITY \\ OREGON STATE UNIVERSITY \\ UNIVERSITY OF OREGON \\ OSAKA UNIVERSITY \\ UNIVERSITY OF SOUTHERN CALIFORNIA
}

\author{
STANFORD UNIVERSITY \\ UNIVERSITY OF TOKYO \\ UNIVERSITY OF UTAH \\ WASHINGTON STATE UNIVERSITY \\ UNIVERSITY OF WASHINGTON \\ * * * \\ AMERICAN MATHEMATICAL SOCIETY \\ CHEVRON RESEARCH CORPORATION \\ NAVAL WEAPONS CENTER
}

The Supporting Institutions listed above contribute to the cost of publication of this Journal, but they are not owners or publishers and have no responsibility for its content or policies.

Mathematical papers intended for publication in the Pacific Journal of Mathematics should be in typed form or offset-reproduced, (not dittoed), double spaced with large margins. Underline Greek letters in red, German in green, and script in blue. The first paragraph or two must be capable of being used separately as a synopsis of the entire paper. The editorial "we" must not be used in the synopsis, and items of the bibliography should not be cited there unless absolutely necessary, in which case they must be identified by author and Journal, rather than by item number. Manuscripts, in duplicate if possible, may be sent to any one of the four editors. Please classify according to the scheme of Math. Rev. Index to Vol. 39. All other communications to the editors should be addressed to the managing editor, Richard Arens, University of California, Los Angeles, California, 90024.

50 reprints are provided free for each article; additional copies may be obtained at cost in multiples of 50 .

The Pacific Journal of Mathematics is published monthly. Effective with Volume 16 the price per volume (3 numbers) is $\$ 8.00$; single issues, $\$ 3.00$. Special price for current issues to individual faculty members of supporting institutions and to individual members of the American Mathematical Society: $\$ 4.00$ per volume; single issues $\$ 1.50$. Back numbers are available.

Subscriptions, orders for back numbers, and changes of address should be sent to Pacific Journal of Mathematics, 103 Highland Boulevard, Berkeley, California, 94708.

PUBLISHED BY PACIFIC JOURNAL OF MATHEMATICS, A NON-PROFIT CORPORATION

Printed at Kokusai Bunken Insatsusha (International Academic Printing Co., Ltd.), 7-17, Fujimi 2-chome, Chiyoda-ku, Tokyo, Japan. 


\section{Pacific Journal of Mathematics}

\section{Vol. 37, No. $2 \quad$ February, 1971}

Charles Compton Alexander, Semi-developable spaces and quotient images of metric spaces .................................... 277

Ram Prakash Bambah and Alan C. Woods, On a problem of Danzer. . . . . . . . . 295

John A. Beekman and Ralph A. Kallman, Gaussian Markov expectations and related integral equations . ....................................

Frank Michael Cholewinski and Deborah Tepper Haimo, Inversion of the Hankel

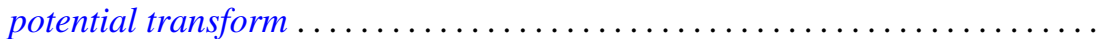

John H. E. Cohn, The diophantine equation

$$
Y(Y+1)(Y+2)(Y+3)=2 X(X+1)(X+2)(X+3) \ldots \ldots \ldots \ldots \ldots
$$

Philip C. Curtis, Jr. and Henrik Stetkaer, A factorization theorem for analytic

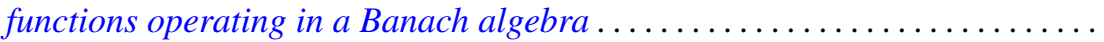

Doyle Otis Cutler and Paul F. Dubois, Generalized final rank for arbitrary limit ordinals

Keith A. Ekblaw, The functions of bounded index as a subspace of a space of entire functions

Dennis Michael Girard, The asymptotic behavior of norms of powers of

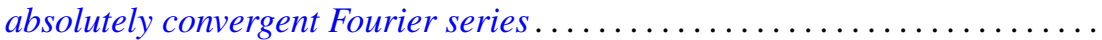

John Gregory, An approximation theory for elliptic quadratic forms on Hilbert spaces: Application to the eigenvalue problem for compact quadratic forms. 383

Paul C. Kainen, Universal coefficient theorems for generalized homology and stable cohomotopy.

Aldo Joram Lazar and James Ronald Retherford, Nuclear spaces, Schauder bases, and Choquet simplexes.

David Lowell Lovelady, Algebraic structure for a set of nonlinear integral operations

John McDonald, Compact convex sets with the equal support property . 429

Forrest Miller, Quasivector topologies

Marion Edward Moore and Arthur Steger, Some results on completability in commutative rings.

A. P. Morse, Taylor's theorem

Richard E. Phillips, Derek J. S. Robinson and James Edward Roseblade, Maximal subgroups and chief factors of certain generalized soluble groups.

Doron Ravdin, On extensions of homeomorphisms to homeomorphisms ...

John William Rosenthal, Relations not determining the structure of $\mathrm{L}$

Prem Lal Sharma, Proximity bases and subbases ........... .

Larry Smith, On ideals in $\Omega_{*}^{u}$. .

Warren R. Wogen, von Neumann algebras generated by operators similar to normal operators

R. Grant Woods, Co-absolutes of remainders of Stone-Čech 